\section{EDUCATION}

Research, Innovation and Solutions on-line ${ }^{(2)}$
PSYCHOLOGY

I+Dti
Electronic Journal of Research

in Educational Psychology

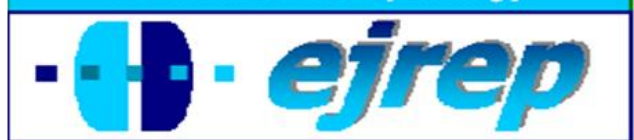

\title{
Creencias del Profesorado de Español sobre los agrupamientos inclusivos del programa de Aulas Temporales de Adaptación Lingüística
}

\section{Antonio J. Rojas Tejada ${ }^{1}$, Pablo Sayans-Jiménez ${ }^{1}$, Raquel M. Cruz del Pino' ${ }^{2}$ y Moshe Tatar}

${ }^{1}$ Departamento de Psicología, Universidad de Almería. Almería.

${ }^{2}$ I.E.S. Santa María del Águila. Delegación de Educación de Almería.

${ }^{3}$ The Hebrew University of Jerusalem. Jerusalén.

España / Israel

Correspondencia: Antonio J. Rojas Tejada. Facultad de Psiclogía. Universidad de Almería. 04120 Almería. España. E-mail: arojas@ual.es 


\section{Resumen}

Introducción. En este trabajo se realiza una evaluación de las creencias sobre los agrupamientos inclusivos del profesorado de español para extranjeros del programa de Aulas Temporales de Adaptación Lingüística (ATAL). El programa ATAL se ha implantado en Andalucía, España, como medida de atención a la diversidad del alumnado inmigrante. Se plantea que los docentes que trabajan directamente con alumnado inmigrante deberán tener unas creencias que sustenten de forma congruente una actuación inclusiva. El objetivo ha sido medir las creencias sobre los agrupamientos inclusivos de este profesorado, mediante una escala creada a tal efecto, y relacionarlas con la forma en cómo realizan su apoyo, dentro o fuera del aula ordinaria, y su experiencia.

Método. Los participantes en este estudio fueron docentes de ATAL de la provincia de Almería. La escala sobre creencias sobre los agrupamientos inclusivos se aplicó a 81 profesores. Resultados. Los resultados avalan la fiabilidad y utilidad de esta medida. El profesorado con puntuaciones altas en creencias inclusivas desarrolla su labor dentro del aula ordinaria con mayor frecuencia que el de puntuaciones bajas. Mientras que el profesorado de creencias altas en los agrupamientos inclusivos valora la labor del entorno y la interacción con los compañeros autóctonos en la resolución de los obstáculos educativos y sociales del alumnado inmigrante. El profesorado con puntuaciones bajas en creencias tiene más años de experiencia y contempla como poco probable que la inclusión del alumnado de ATAL en el aula ordinaria mejore sus competencias lingüísticas y académicas, así como su inclusión curricular y social.

Discusión y Conclusión. Existen dos grupos de profesorado, uno, que ve poco probable que la inclusión del alumnado de ATAL en el aula ordinaria mejore sus competencias linguiísticas y académicas, así como su inclusión curricular y social. El profesorado que sostiene este tipo de creencias, es partidario de pocas o ninguna intervención y cree que la heterogeneidad de las clases le ha sido impuesta. Y otro grupo que asume que los problemas de aprendizaje del alumnado provienen, en mayor medida, del entorno educativo. Este conjunto de creencias coinciden con las del profesorado de ATAL que vincula en mayor medida la inclusión del alumnado del programa en el aula ordinaria con mejoras en competencias lingüísticas, en rendimiento académico y en las relaciones sociales.

Palabras Clave: Inclusividad, Creencias inclusivas, Aulas Temporales de Adaptación Lingüística, profesorado de español para inmigrantes, inmigrante. 


\section{How Teachers of Spanish Feel about Inclusive Groupings in the Temporary Linguistic Adaptation Classrooms Abstract}

Introduction. The aim of this paper is to assess Spanish as a Foreign Language Teachers' beliefs about inclusive groupings in Temporary Classroom of Linguistic Adaptation program (TCLA program). TCLA program has been implemented in Andalusia, Spain, as a measure of attention to the diversity of immigrant students. It is suggested that teachers who work directly with immigrant students must have a beliefs that support inclusive teaching consistently. The purpose has been to measure the inclusive beliefs of TCLA program teachers using a scale created for this purpose, and relate to the way how they do their support, inside or outside the ordinary classroom, and their years of experience.

Method. Participants in this study were TCLA teachers of the province of Almeria. The scale on beliefs about inclusive grouping was applied to 81 teachers.

Results. The results support the reliability and validity of this measure. Teachers with high scores in inclusive beliefs develop their work within the regular classroom more frequently than low scores. Teachers with high-believes about inclusive groupings appreciate the environment and interaction with native partners as keys to work in solving social and educational immigrant students' barriers. Teachers with low scores on beliefs about inclusive groupings have more years of experience and see as unlikely that the inclusion of TCLA students in regular classroom improve their language and academic skills, as well as their curricular and social inclusion.

Discussion and Conclusion. There are two groups of teachers, one group think is very unlikely that the inclusion of TCLA students in regular classroom improve their language skills and academic and curricular and social inclusion. Teachers holding these beliefs think that little or no intervention is good and the heterogeneity of the classes has been imposed. A second group assumes that the learning problems of students come to a greater extent, the educational environment. This set of beliefs coincide with those of TCLA teachers linking further the inclusion of the program students in regular classrooms with improved language skills, academic performance and social relationships.

Keywords: Inclusivity, Inclusive Beliefs, Temporary Classrooms of Linguistic Adaptation, SSL teacher, immigrant. 


\section{Introducción}

En líneas generales, la educación inclusiva pretende dar respuesta a las necesidades educativas de todo el alumnado de un centro escolar, empleando procedimientos que aumenten la participación de estos estudiantes y reduzcan su exclusión (Booth y Ainscow, 2002), aunque la educación inclusiva es un concepto abierto a diferentes interpretaciones y apreciaciones. Esta propuesta inclusiva implica proporcionar apoyo dentro del aula ordinaria al Alumnado con Necesidad Específica de Apoyo Educativo (ANEAE), “entendiendo por tal el alumnado con necesidades educativas especiales, el que se incorpore de forma tardía al sistema educativo, el que precise de acciones de carácter compensatorio y el que presente altas capacidades intelectuales" (Orden de 25 de julio de 2008, B.O.J.A. núm. 167, pp. 7), entre otros aspectos. Este ANEAE debe estar incluido en los mismos entornos educativos que el resto de sus compañeros de clase y participar del mismo currículum.

Para la incorporación de los ANEAE en el aula ordinaria, necesariamente el sistema educativo tiene que afrontar replanteamientos institucionales (Jurado y Ramírez, 2009). En la actualidad, la legislación educativa se hace eco sobradamente de las aportaciones propuestas por la UNESCO, tanto en la Declaración de Salamanca (1994) como en las Directrices para la Inclusión (2005), en lo que respecta a atención a la diversidad e inclusividad. Sin embargo, las imposiciones normativas verticales por parte de la administración no siempre coinciden con las ideas sobre inclusividad que residen en la comunidad educativa (Marchant, 2009).

Existen diferentes programas que pretenden eliminar o minimizar cualquier barrera $\mathrm{u}$ obstáculo que impida la participación del ANEAE en el mismo currículum ordinario y con su grupo de iguales. Uno de ellos consiste en reforzar la actividad docente con profesorado especialista (p.e. docentes de español como segunda lengua, de apoyo, de apoyo a la integración, de educación especial o de compensatoria). Estos docentes especialistas son los responsables de apoyar el desarrollo integral del los ANEAE eliminando las barreras que impidan su aprendizaje (Jurado y Ramírez, 2009). Este trabajo se centra en este profesorado de español para extranjeros, concretamente del profesorado que participa en el programa de Aulas Temporales de Adaptación Lingüística (ATAL) que se desarrolla en Andalucía (España). Este programa pretende la enseñanza-aprendizaje del español como lengua vehicular para alumnado inmigrante (considerado ANEAE), vinculado a profesorado específico. 
La legislación vigente indica que el apoyo del programa ATAL debe realizarse dentro del aula ordinara, pudiendo llevarse a cabo fuera, cuando las circunstancias especiales en la comprensión de la lengua así lo aconsejen (Orden de 15 de enero de 2007, BOJA núm. 33, pp. 7-11). Es decir, no se debe realizar agrupamientos segregadores, que excluyan al alumnado inmigrante del resto de sus compañeros. El programa ATAL pretende que la intervención se realice mediante un agrupamiento inclusivo, dentro del aula ordinaria. Los objetivos principales de este programa son dos: 1) facilitar la atención específica de alumnado inmigrante con desconocimiento del español con un programa que apoye la adquisición de competencias lingüísticas y comunicativas, y, 2) permitir la integración de este alumnado en el entorno social y escolar en el menor tiempo posible y con garantías de progreso en el aula ordinaria.

En Andalucía, la aparición de las ATAL como medida de atención a la diversidad del alumnado inmigrante supone el surgimiento de un recurso educativo que se aplica de diferentes formas, tanto en las distintas provincias andaluzas (Ortiz, 2007) como en los centros. Esta flexibilidad de adaptación sería una gran virtud del programa ATAL, de no ser porque algunas de las actuaciones se están tornando discriminatorias, entre otros aspectos por la libre interpretación de la normativa (Ortiz, 2007). De hecho, el apoyo al alumnado dentro de este programa corresponde fundamentalmente a los docentes ATAL. El programa está enfocado para desarrollarse dentro del aula ordinaria, sin embargo, es habitual que el alumnado reciba apoyo adicional en un aula específica a lo largo de las horas lectivas en el centro. No es nada inusual que el profesorado ATAL trabaje directamente con el alumnado inmigrante de forma individual o en grupos pequeños (Rojas, Cruz, Sayans y Tatar, 2011). Es decir, existen diferentes tipos de actuación del Profesor de ATAL aplicando el mismo programa.

De esta forma, el esfuerzo educativo se orienta a la consecución de objetivos generales de enseñanza mínimos y se aleja de la preocupación por una educación inclusiva acorde con las capacidades de cada estudiante. Por lo que las "circunstancias especiales" que deberían darse para aplicar el programa ATAL fuera del aula ordinaria, suelen ser empleadas como justificante más veces de las necesarias (Ortiz, 2007). Sin embargo, el fomento de prácticas escolares que segreguen al alumnado en "grupos especiales" perjudica su desarrollo al separarlos de su entorno real y no incluirlos en un proyecto educativo común (Jurado y Ramírez 2009). 
Para incrementar el interés o la motivación del alumnado para la adquisición de una segunda lengua se han de promover procesos que favorezcan la integración y la percepción de pertenencia al grupo mayoritario, lo que permitirá la mejora de competencias tanto lingüístico-comunicativa como lingüístico-académica (Linares, 2007). En el caso del aprendizaje de idiomas, parece que el mejor modelo lingüístico para los estudiantes menos competentes sería el propio grupo de iguales nativos (Coelho, 2006), lo cual supone también para éstos últimos la mejora de su conciencia metalingüística. Aprender con el grupo de iguales es más fácil, entretenido, y a menudo, efectivo (Hopkins, West y Ainscow, 1996). Los grupos ofrecen apoyo social para alcanzar el éxito académico que no se obtendría de otra forma. El aula ordinaria es el lugar idóneo para aprender y desarrollar la lengua, ya que este contexto es en el que el alumnado recién llegado puede regular sus intercambios con el resto del alumnado y con el profesorado desde la nueva lengua (Vila et al., 2009).

\section{Creencias sobre agrupamientos inclusivos de las ATAL}

Un elemento clave para el éxito de la implementación de políticas educativas es el punto de vista del personal que va a tener la responsabilidad de llevarlo a cabo, es decir, el propio profesorado (Avramidis y Norwich, 2002). Las concepciones y creencias del propio profesorado sobre los procesos de escolarización son muy importantes y constituyen una de las dimensiones claves en la implementación de programas educativos (López, Echeita y Martín, 2009). Aunque hay intentos en la literatura de establecer las diferencias entre concepciones y creencias, ambos términos suelen usarse de forma indistinta (García, Azcárate y Moreno, 2006). Mientras las creencias del profesor son ideas poco elaboradas, generales o específicas, las cuales forman parte del conocimiento que posee el docente pero carecen de rigor para mantenerlas e influyen de manera directa en su desempeño, las concepciones del profesor consisten en la estructura que cada profesor da a sus conocimientos para posteriormente enseñarlos o transmitirlos a sus estudiantes (García, Azcárate y Moreno, 2006). Las creencias están relacionadas con el conocimiento o información de la que se dispone sobre el objeto de las mismas, y se podrían conceptualizar como la probabilidad subjetiva de que un evento o situación ocurra (Wyer y Albarracín, 2005). Para este estudio, las creencias se operativizan como la probabilidad subjetiva de que una proposición sea cierta (Wyer y Albarracín, 2005). Más concretamente en, este trabajo se abordarán las creencias comportamentales referidas a la agrupación inclusiva del alumnado inmigrante dentro del aula ordinaria. Éstas representan la probabilidad percibida de que un comportamiento concreto (inclusión del alumnado inmigran- 
te en el aula ordinaria) se asocie con unos determinados resultados (relacionados con la competencia lingüística, el rendimiento académico y las relaciones interpersonales), tal y como podría derivarse de la Teoría de la Acción Planeada (p.e. Azjen, 1991; Azjen y Fishbein, 2005).

En este trabajo nos centraremos en las creencias que el profesorado ATAL tiene sobre los agrupamientos inclusivos del programa ATAL, ya que estas mismas pueden ser determinantes para el éxito del programa. Es necesario considerar que, al menos, los docentes que trabajan directamente con alumnado inmigrante tengan unas creencias que sustenten de forma congruente su actuación. Como aclara Gibbs (2007), el desarrollo de prácticas inclusivas es incompatible con creencias segregadoras. Se trata de explorar en qué grado el profesorado ATAL considera que incluir al alumnado inmigrante dentro del aula ordinaria puede estar asociado a las ventajas de la inclusividad del programa: mejor adquisición de competencias lingüísticas, mejora de los resultados académicos y mejor integración.

Poca certeza (creencias bajas) sobre la asociación entre las ventajas del programa y los agrupamientos inclusivos del programa ATAL, implicaría que no se percibe como influyente, en el éxito en la educación del alumnado inmigrante, que éste trabaje con el resto de compañeros en el aula ordinaria, con el apoyo de profesorado específico. Este tipo de creencias probablemente se sustenten sobre la idea de que los problemas del alumnado inmigrante del programa son sólo relativos al propio alumnado. Siguiendo esta línea, el entorno educativo y las interacciones entre iguales y con los docentes no tendrían mucho peso en la mejora del resultado del alumnado inmigrante. Por otro lado, creencias altas (o certeza sobre la asociación entre las ventajas del programa y los agrupamientos inclusivos del programa ATAL) supondrían darle un mayor peso al entorno educativo y las relaciones del alumnado inmigrante, no sólo para el aprendizaje del idioma, sino para su inclusión curricular y social. En este sentido, también puede resultar de especial relevancia mostrar en qué forma podrían relacionarse las creencias del profesorado de las ATAL con la manera en que éste aplica el programa, es decir, en función del tipo de actuación que lleva a cabo en el centro.

En los últimos tiempos se han desarrollado diversas escalas para evaluar las creencias y actitudes del profesorado hacia la inclusividad (Cochran, 1997; Mahat, 2008; Wilczenski, 1992, 1995). En la mayoría de los estudios, actitudes y creencias hacia la inclusividad está referida principalmente a alumnado cuyas necesidades educativas afectan al aprendizaje de 
forma permanente. Sin embargo, la inclusividad de ANEAE también afecta al alumnado inmigrante con desconocimiento del español, como es el caso del alumnado de ATAL, cuya necesidad educativa es temporal.

\section{Objetivos}

En esta investigación se utiliza una escala desarrollada específicamente para evaluar las creencias del profesorado de ATAL sobre agrupamientos inclusivos del programa ATAL. El objetivo de este trabajo es analizar los resultados sobre las creencias acerca de los agrupamientos inclusivos que tiene el profesorado ATAL en el ámbito de su trabajo a partir de los datos proporcionados por dicha escala. Además, se pretende conocer las creencias del profesorado de ATAL sobre los agrupamientos inclusivos del programa según el tipo de actuación de este profesorado. Es decir, se intentará comprobar si existe consistencia entre estas creencias y el tipo de actuación que lleva a cabo dicho profesorado (dentro o fuera del aula ordinaria). Finalmente, se tratará de conocer la relación con otras variables sociodemográficas del profesorado ATAL (como años de experiencia en el programa de ATAL y edad del profesorado). En la revisión llevada a cabo por Avramidis y Norwich (2002) queda constancia de que el tiempo de experiencia en el puesto es una de las variables relevantes estudiadas en la investigación sobre actitudes hacia la inclusión y la integración.

\section{Método}

\section{Participantes}

Los participantes en este estudio fueron docentes de ATAL (81 de un total de 84) de la provincia de Almería (10 hombres y 71 mujeres) que ejercían en 132 centros educativos (45 en primaria y 87 en secundaria), caracterizados por una alta tasa de inmigrantes (un promedio de $10 \%$ para el total de la población). Los años de experiencia del profesorado en el programa de ATAL oscilaban entre 1 y 10 años (Media=2.8 años; DT=2.3). Todos los centros educativos con programa de ATAL fueron incluidos en el estudio. El total de profesorado de ATAL en el programa en Almería en el curso 2009/2010 era de 84.

\section{Variables e Instrumentos}

Creencias sobre agrupamientos inclusivos de las ATAL. Se elaboró una escala expresamente para esta investigación donde las creencias se operativizaron en tres contenidos concretos: competencia lingüística, rendimiento académico y relaciones interpersonales. La esca- 
la final, tras pasar por las etapas habituales de construcción de tests, resultó compuesta por 10 ítems: dos ítems sobre competencia lingüística, cuatro ítems sobre rendimiento académico y cuatro ítems sobre relaciones interpersonales (ver cuestionario en anexo I). Los participantes debían responder indicando su grado de acuerdo respecto a los ítems. Las categorías de respuesta de los ítems iban desde 1 (totalmente en desacuerdo) a 5 (totalmente de acuerdo). Todos los ítems estaban redactados en positivo, salvo el ítem cuatro que lo estaba en negativo (y se invierte su puntuación).

Tipo de actuación del Profesor de ATAL. Una pregunta se utilizó para conocer dónde llevaba a cabo el programa de ATAL (¿Ha desarrollado su trabajo como docente de ATAL dentro del aula ordinaria?). Las respuestas a esta pregunta se clasificaron en: 1) Siempre; 2) Varias veces al mes o al trimestre; 3) Alguna vez durante el curso o nunca.

Otras Variables. Se preguntaron otras cuestiones tales como años de experiencia como profesorado ATAL, así como sexo y edad.

\section{Procedimiento}

Dado que las creencias pueden estar referidas a eventos concretos o situaciones más generales (Fabrigar, Macdonald y Wegener, 2005) la aplicación del cuestionario, que contenía todas las escalas y preguntas, se administró de forma colectiva y se llevó a cabo tras una sesión de grupal de todo el profesorado del programa. En esta sesión se trataba de forma explícita la necesidad de cumplir con la normativa de incluir al alumnado inmigrante en el aula ordinaria. De esta forma, se espera que la sesión grupal sirviese para la activación previa del conocimiento concreto sobre el que se basan este tipo de creencias. Todos los profesores respondieron voluntariamente a las preguntas. El cuestionario era anónimo.

\section{Análisis de datos}

Para la descripción de los valores del test se han empleado estadísticos descriptivos (media y desviación típica). El estudio de la estructura interna del test se ha llevado a cabo a través de un Análisis Factorial Exploratorio de componentes principales con rotación oblimin. Previamente se efectuaron las pruebas KMO y la de esfericidad de Bartlett. Para el análisis de diferencias de las medias de creencias de los grupos en función de que manifiesten haber desarrollado su labor en la clase ordinaria o no, se ha realizado una ANOVA unifactorial, donde se han hecho comparaciones a posteriori con la prueba de Sheffé. Igualmente para constrastar 
las diferencias de las medias de creencias en los grupos en función del tiempo que llevan como profesorado de ATAL se ha realizado una ANOVA unifactorial, con comparaciones a posteriori con la prueba de Sheffé. Se han utilizado diagramas de Caja y Bigotes para representar gráficamente las puntuaciones de creencias inclusivas en función de las variables de tipo de actuación de Profesorado ATAL y años de experiencia.

\section{Resultados}

Propiedades Psicométricas de la Escala de Creencias sobre agrupamientos inclusivos de las ATAL

Los valores de fiabilidad estimada del cuestionario han sido adecuados. Los resultados mediante el coeficiente alpha de Cronbach dieron un valor de .85 y mediante el procedimiento de dos mitades (pares e impares) fue de .91 (con la corrección de Spearman-Brown). Para el estudio de la estructura interna del test se ha llevado a cabo un Análisis Factorial Exploratorio de componentes principales con rotación oblimin. Previamente a la interpretación del análisis factorial se aplicaron las pruebas KMO y la prueba de esfericidad de Bartlett. Los valores de KMO se mostraron aceptables con un valor de .763, la esfericidad se mostró estadísticamente significativa (tabla 1), con lo que la matriz de datos es factorizable.

Tabla 1. Estadísticos descriptivos y Análisis Factorial del Cuestionario de Creencias Inclusivas (cargas factoriales, comunalidades, varianza explicada y pruebas KMO y de esfericidad de Barlett)

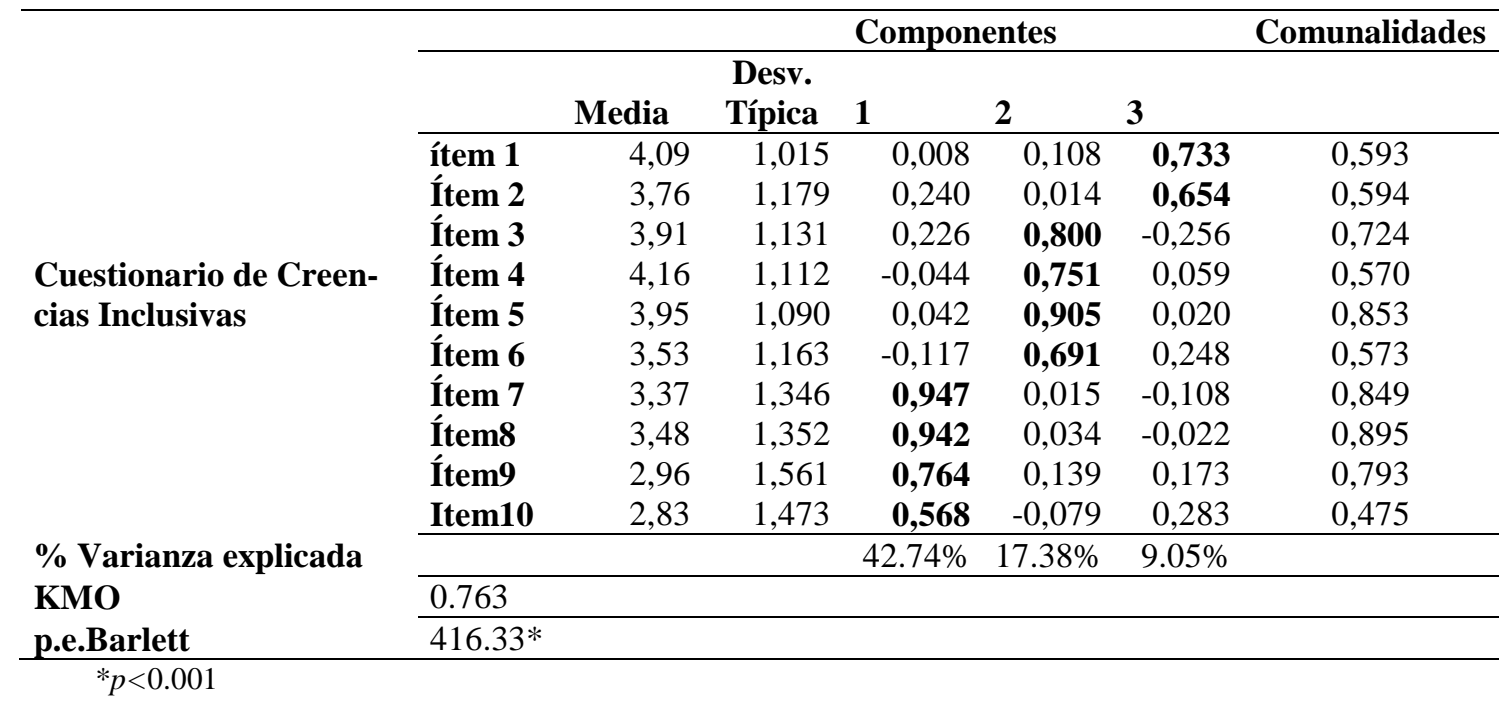

En la determinación de los elementos de cada factor se consideró la regla habitual de saturaciones mínimas .35-.40 como los niveles mínimos para considerar que un elemento es 
un indicador del factor. Como puede apreciarse en la tabla 1, aparece una solución de tres factores que tienen una interpretación coherente con la propuesta teórica. Los tres factores claramente separan los ítems de competencia lingüística, los de rendimiento académico y los de relaciones interpersonales. Los tres factores acumulan aproximadamente el $69.18 \%$ de la varianza explicada. Estos resultados muestran evidencias de unidimensionalidad según el criterio de considerar que el primer factor tenga más de un $40 \%$ de varianza explicada (Carmines y Zeller, 1979) y el criterio de que la diferencia de varianza explicada entre el primer factor y el segundo es el triple que la diferencia de varianza explicada entre el segundo y el tercero (Hatti, 1985). Estos hallazgos muestran evidencias empíricas de validez de la propuesta teórica de la medida del constructo.

Creencias sobre agrupamientos inclusivos de las ATAL del Profesorado y relación con otras variables.

La medida de creencias sobre agrupamientos inclusivos de las ATAL se ha obtenido mediante la media aritmética de las respuestas a las diez preguntas de la escala (sólo para el ítem 4 se ha invertido la puntuación). Los resultados muestran una media 3.601 (DT=0.820) (recordemos que las puntuaciones podían oscilar entre la mínima de 1 y la máxima de 5). Al comparar las medias de creencias en los grupos en función de que manifiesten haber desarrollado su labor en la clase ordinaria o no, se he encontrado que el grupo con mayores puntuaciones en el test de creencias es el que ha manifestado entrar siempre en el aula ordinaria para realizar su trabajo (media=4.088; DT=0.786), frente al que menores puntuaciones en creencias sobre agrupamientos inclusivos de las ATAL tiene, que es el grupo que ha manifestado no entrar nunca o solo alguna vez en el curso dentro de clase ordinaria (media=3.310; DT= 0.674), pasando por el grupo intermedio, que manifestaba entrar varias veces al mes/trimestre dentro de clase ordinaria (media $=3.645$; DT $=0.778$ ). Al realizar una ANOVA, una vez probada la normalidad y la homogenidad de las varianzas de las puntuaciones de cada grupo, encontramos que las diferencias en las medias de los grupos son estadísticamente significativas $\left(F_{2,75}=4.489 ; p<.015\right)$. Al realizar las comparaciones a posteriori con la prueba de Sheffé, encontramos diferencias estadísticamente significativas entre el grupo que entra siempre en el aula ordinaria para realizar su trabajo y el grupo que no entra nunca o solo alguna vez en el curso dentro de clase ordinaria $(p<.016)$. Ver Tabla 2. 
Tabla 2. Estadísticos descriptivos de la Escala y Análisis de Varianza.

\begin{tabular}{|c|c|c|c|c|c|}
\hline $\begin{array}{l}\text { Desarrollo de la labor de profesorado de ATAL DESCRIP- } \\
\text { TIVOS }\end{array}$ & $\mathrm{N}$ & \multicolumn{2}{|c|}{ Media } & \multicolumn{2}{|c|}{$\begin{array}{l}\text { Desviación } \\
\text { típica }\end{array}$} \\
\hline Siempre dentro de Clase Ordinaria & 16 & \multicolumn{2}{|c|}{4.088} & \multicolumn{2}{|l|}{0.786} \\
\hline Varias veces al mes/trimestre dentro de Clase Ordinaria & 44 & \multicolumn{2}{|c|}{3.645} & \multicolumn{2}{|l|}{0.778} \\
\hline Alguna vez en el curso o nunca dentro de Clase Ordinaria & 18 & \multicolumn{2}{|c|}{3.310} & \multicolumn{2}{|l|}{0.674} \\
\hline Total & 78 & \multicolumn{2}{|c|}{3.658} & \multicolumn{2}{|l|}{0.791} \\
\hline ANOVA & $\begin{array}{l}\text { Suma de } \\
\text { cuadrados }\end{array}$ & gl & $\begin{array}{l}\text { Media } \\
\text { cuadrática }\end{array}$ & $\mathrm{F}$ & Sig. \\
\hline Inter-grupos & 5.148 & 2 & 2.574 & 4.489 & 0.014 \\
\hline Intra-grupos & 43.010 & 75 & 0.573 & & \\
\hline Total & 48.159 & 77 & & & \\
\hline $\begin{array}{l}\text { Años de experiencia como profesorado ATAL DESCRIP- } \\
\text { TIVOS }\end{array}$ & $\mathrm{N}$ & \multicolumn{2}{|c|}{ Media } & \multicolumn{2}{|c|}{$\begin{array}{l}\text { Desviación } \\
\text { típica }\end{array}$} \\
\hline De 0 hasta 2 años & 47 & \multicolumn{2}{|c|}{3.684} & \multicolumn{2}{|l|}{0.745} \\
\hline De 3 hasta 4 años & 13 & \multicolumn{2}{|c|}{3.951} & \multicolumn{2}{|l|}{0.873} \\
\hline Más de 4 años & 21 & \multicolumn{2}{|c|}{3.219} & \multicolumn{2}{|l|}{0.842} \\
\hline Total & 81 & \multicolumn{2}{|c|}{3.607} & \multicolumn{2}{|l|}{0.820} \\
\hline ANOVA & $\begin{array}{l}\text { Suma de } \\
\text { cuadrados }\end{array}$ & $\mathrm{gl}$ & $\begin{array}{l}\text { Media } \\
\text { cuadrática }\end{array}$ & $\mathrm{F}$ & Sig. \\
\hline Inter-grupos & 4.983 & 2 & 2.492 & \multirow[t]{3}{*}{3.978} & \multirow[t]{3}{*}{0.023} \\
\hline Intra-grupos & 48.856 & 78 & 0.626 & & \\
\hline Total & 53.839 & 80 & & & \\
\hline
\end{tabular}

Al comparar las medias de creencias en los grupos en función del tiempo que llevan como profesorado de ATAL, encontramos que el grupo con mayores puntuaciones en creencias sobre agrupamientos inclusivos de las ATAL es el que lleva en el puesto entre 2 y 4 años de antigüedad (media=3.951; DT=0.873), frente al grupo de más de 4 años trabajando como ATAL que muestra menores puntuaciones (media=3.219; DT= 0.842). El grupo de menos de 2 años de antigüedad tuvo una media de 3.684 (DT= 0.745). Una vez comprobada la normalidad y la homogenidad de las varianzas de cada grupo, el ANOVA mostró diferencias estadísticamente significativas $\left(\mathrm{F}_{2,78}=3.978 ; p<.023\right)$ entre estas medias grupales. En este caso, las comparaciones a posteriori con la prueba de Sheffé, mostraron diferencias significativas estadísticamente significativas entre el grupo de más de 4 años y el de 2 a 4 años ( $p<.037)$. No se encontraron correlaciones estadísticamente significativas con la edad $(\mathrm{r}=-.174 ; \mathrm{p}=.145)$. Ver Figura 1. 


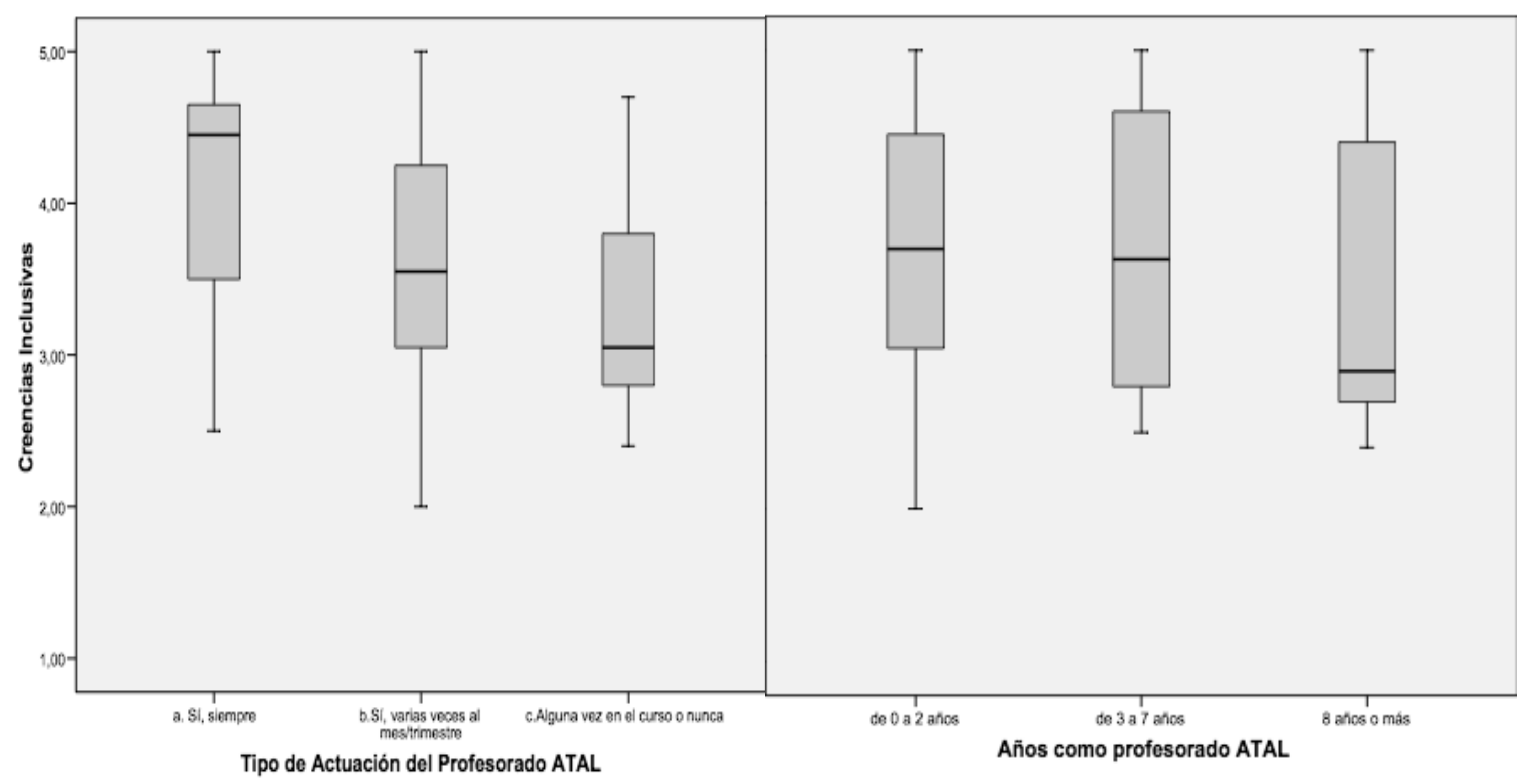

Figura 1. Diagramas de Caja y Bigotes de Creencias por Tipo de actuación de Profesorado ATAL y por años de Experiencia ATAL.

\section{Discusión y conclusiones}

Este trabajo se ha centrado en la medición de las creencias sobre los agrupamientos inclusivos del profesorado de español para extranjeros en un programa de adaptación lingüística de alumnado inmigrante en España. Las creencias inclusivas se han considerado importantes debido a que, por un lado, son un componente fundamental para el éxito de la educación inclusiva (Avramidis y Norwich, 2002; Gibbs, 2007; Stanovitch y Jordan, 1998), y, por otro lado, porque, para que se pueda desarrollar la inclusividad en el aula, es esencial compartir visiones parecidas del proceso para que se pueda dar una buena colaboración entre docentes (Boudah y Hasbrouck, 1998).

Para la medida de estas creencias inclusivas en esta investigación se ha elaborado una escala específica para el profesorado del programa ATAL. Esta escala ha mostrado ser una medida fiable, breve y útil para evaluar dichas creencias. Los resultados obtenidos avalan la utilidad de esta herramienta no sólo para la medición de creencias, sino también para comprobar la consistencia entre creencias inclusivas y otras variables como el tipo de actuación del Profesorado de ATAL (p.e. desarrollar el programa dentro o fuera de la clase ordinaria) y la relación con la experiencia como profesorado de ATAL. 
En general, la puntuación promedio del grupo sugiere un nivel medio de creencias del profesorado ATAL sobre los agrupamientos inclusivos del programa. El profesorado con resultados más altos en creencias sobre agrupamientos inclusivos de las ATAL manifiesta que desarrolla su labor dentro del aula ordinaria con mayor frecuencia que el profesorado con puntuaciones más bajas. Estas diferencias muestran dos tendencias evaluativas completamente diferentes hacia la inclusión del alumnado del programa ATAL dentro del aula ordinaria. Se debe hacer notar la similitud de estas dos visiones de la intervención con el alumnado ATAL, con las creencias que puede mantener el profesorado hacia la inclusión de alumnado con necesidades educativas especiales (este alumnado también se encuentra dentro de la categoría estatal ANEAE, pero sus necesidades vendrían derivadas de discapacidad o trastornos graves de conducta).

Por un lado, se encuentra el profesorado que contempla como poco probable que la inclusión del alumnado de ATAL en el aula ordinaria mejore sus competencias lingüísticas y académicas, así como su inclusión curricular y social. Las creencias de este profesorado se asemejan al conjunto de creencias etiquetado por Stanovich y Jordan (1998) como patognómico. El profesorado que sostiene este tipo de creencias, es partidario de pocas o ninguna intervención y cree que la heterogeneidad de las clases le ha sido impuesta, además de entender que las intervenciones del sistema deben dirigirse a reducir ésta heterogeneidad (Stanovich y Jordan, 1998).

Por otro lado, estas autoras etiquetan otro conjunto de creencias como intervencionista (Stanovich y Jordan, 1998). El profesorado con este conjunto de creencias asume que los problemas de aprendizaje del alumnado provienen, en mayor medida, del entorno educativo. Este conjunto de creencias coinciden con las del profesorado de ATAL que vincula en mayor medida la inclusión del alumnado del programa en el aula ordinaria con mejoras en competencias lingüísticas, en rendimiento académico y en las relaciones sociales. Las puntuaciones obtenidas en creencias inclusivas por el profesorado ATAL puestas en relación con los años de experiencia en el programa indican que a partir de los cuatro años de experiencia, el profesorado puntúa más bajo en la escala de creencias inclusivas.

Respecto al tiempo de experiencia en el puesto, algunos de estos estudios muestran concordancia con los resultados obtenidos en este trabajo, en los que se constata la disminu- 
ción de creencias inclusivas a partir de ciertos años en el puesto (Avramidis y Norwich, 2002). El número de años de desempeño en el programa ATAL puede ser un factor que contribuya a la disminución de las creencias inclusivas debido a las características y a la forma en cómo se desempeña el puesto. Avramidis y Norwich (2002) concluyen en su estudio que, debido a los factores de estrés, la experiencia con alumnado con necesidades de apoyo podría no favorecer la aceptación de la inclusión. Esta variable ha de ser tenida en cuenta para la elaboración de perfiles del profesorado que se adecúen al programa.

Por otro lado, aunque de forma tangencial, los resultados ponen de manifiesto que parte de los docentes ATAL no cumplen la norma de desarrollar el programa dentro del aula, cuya única excepción deberían ser las circunstancias especiales en la compresión de la lengua. Este hecho pone de manifiesto la necesidad de regular sin ambages y de una forma más coherente el modelo educativo que se quiere implantar en los centros educativos andaluces. Los mismos textos legislativos que regulan aspectos educativos que abogan por un modelo educativo inclusivo, permiten y promueven luego prácticas totalmente contrarias, como pueden ser los agrupamientos flexibles para la atención al alumnado en un grupo específico (Orden de 25 de julio de 2008, B.O.J.A. núm. 167, pp. 8-9).

Finalmente y con el ánimo de mejora de cualquier programa de adaptación lingüística es importante señalar la importancia que, para la eficacia de los procesos de inclusión, tiene el desarrollo de programas de formación de los profesionales que van a llevar a cabo la educación inclusiva. Es necesario realizar investigaciones sobre la forma en que los docentes planifican y llevan a cabo la inclusión en el aula ordinaria; de esta forma, se contribuirá a desarrollar perfiles docentes con características predictivas del éxito de la inclusividad. Y en este sentido, sería recomendable añadir a los criterios de selección del profesorado, por ser un puesto específico, determinadas características que ayuden a discriminar entre perfiles con un mayor nivel de adecuación al programa (p.e. aquellos que tengan un mayor nivel de creencias inclusivas). Por último, será importante en el futuro la aplicación de medidas como las de este trabajo en los distintos programas en los que se promueven agrupamientos inclusivos de alumnado inmigrante, tanto para el profesorado de apoyo, como para el profesorado del aula ordinaria. Y como no, investigar también las condiciones organizacionales que pueden fomentar y facilitar la inclusión de los inmigrantes. 


\section{Agradecimientos}

Este trabajo ha sido posible gracias a un Proyecto de Investigación de Excelencia financiado por la Consejería de Economía, Innovación y Ciencia de la Junta de Andalucía (Ref. P09-SEJ-4657) y cofinanciado por Fondos FEDER, a la colaboración del Equipo Técnico Provincial de Orientación Educativa y Profesional de la Delegación Provincial de Educación de Almería y del profesorado de ATAL.

\section{Referencias}

Avramidis, E. y Norwich, B. (2002). Teachers' attitudes towards integration/inclusion: a review of the literature. European Journal of Special Needs Education, 17(2), 129-147.

Azjen, I (1991). Theory of planned behavior. Organizational behavior and human decision processes, 50, 179-211.

Azjen, I y Fishbein, M. (2005). The influence of attitudes on behavior. En D. Albarracín, B.T. Johnson y M.P Zanna (eds). The handbook of attitudes (pp.173-221). Mahwah, NJ: Erlbaum.

Booth, T. y Ainscow, M. (2002). Index for inclusion: Developing learning and participation in schools (Rev. ed.). Bristol: CSIE.

Boudah, D. J. y Hasbrouck, J. E. (1998). Creating meaningful change in the practice of inclusion: Barriers and solutions. National FORUM of Educational Administration and Supervision Journal-Electronic, 15E (4), 62-68. www.nationalforum.com.

Carbonell, F. (2002). Las dificultades en la integración de los alumnos inmigrantes: síntomas y causas. Propuestas de mejora. II Jornadas sobre Interculturalidad en la Región de Murcia. Murcia: Universidad de Murcia.

Carmines, E. y Zeller, R. (1979). Reliability and validity assessment. California, Sage.

Coelho, E. (2006). Enseñar y aprender en escuelas multiculturales. Una aproximación integrada. Barcelona: I.C.E.

Cochran, H. K. (1997). The development and psychometric analysis of the Scale of Teachers' Attitudes Toward Inclusion (STATIC). Paper presented at the Annual meeting of the Mid-South Educational Research Association, Memphis, Tennessee.

Fabrigar, L.R., Macdonald, T.K. y Wegener, D.T. (2005). The Structure of Attitudes. En Albarracín, D., Johnson, B.T. y Zanna, M.P. (eds). The handbook of attitude (pp.79-124). Mahwah, NJ: Erlbaum. 
García, L., Azcárate, C. y Moreno, M. (2006). Creencias, concepciones y conocimiento profesional de profesores que ensenan cálculo diferencial a estudiantes de ciencias económicas. Revista Latinoamericana de Investigación en Matemática Educativa, 9(1), 85116.

Gibbs, S. (2007). Teachers` perceptions of efficacy: beliefs that may support inclusion or segregation. Educational \&Child Psychology, 24(3), 47-53.

Hattie, J. (1985). Methodology review assessing unidimensionality of tests and items. Applied Psychological Measurement, 9 (2), 139-164.

Hopkins, D., West, M. y Ainscow, M. (1996). Improving the Quality of Education for All. London: David Fulton.

Jacobo H., Loubet R. y Armenta M. (2010). La formación de educadores de migrantes: una perspectiva compleja. Revista Latinoamericana de Inclusión Educativa, 4(1), 45-63.

Jurado, P. y Ramírez, A. (2009). Educación inclusiva e interculturalidad en contextos de migración. Revista Latinoamericana de Educación Inclusiva, 3(2), 109-124.

Linares, J. (2007). Programas y modelos de trabajo con niños y jóvenes inmigrantes en la enseñanza de español El2. Lingüística en la Red, 5. Monográfico. La enseñanza de segundas lenguas a inmigrantes. http://linred.com/numero5/html

López, M., Echeita, G. y Martín, E. (2009). Concepciones sobre el proceso de inclusión educativa con alumnos con discapacidad educativa en la educación secundaria obligatoria. Cultura y Educación, 21(4), 485-496.

Mahat, M. (2008). The development of a psychometrically-sound instrument to Measure teachers' multidimensional attitudes toward inclusive education. International Journal of Special Education, 23(1), 82-92.

Marchant, C. (2009). Sobre reformas, integración-inclusión y exclusión educativa: Reflexiones a partir de la experiencia española. Revista Latinoamericana de Educación Inclusiva, 3(2), 15-25.

Orden de 15 de enero de 2007, por la que se regulan las medidas y actuaciones a desarrollar para la atención del alumnado inmigrante y, especialmente, las Aulas Temporales de Adaptación Lingüística. Boletín Oficial de la Junta de Andalucía, 14 de febrero de 2007, núm. 33, pp. 7-11.

Orden de 25 de julio de 2008, por la que se regula la atención a la diversidad del alumnado que cursa la educación básica en los centros docentes públicos de Andalucía. Boletín Oficial de la Junta de Andalucía, 22 de agosto de 2008, núm. 167, pp. 7-14. 
Ortiz, M. (2007). Segunda lengua como medio de integración escolar. Revista Iberoamericana de Educación, 41(4), 1-5.

Rojas, A.J., Cruz, R.M., Sayans, P. y Tatar, M. ( 2011). El profesorado del programa ATAL: ¿innovador o tradicional? Comunicación presentada en el I Congreso Internacional sobre las Migraciones en Andalucía, 16-18 febrero, 2011, Granada, España.

Stanovitch, P.J. y Jordan, A. (1998). Canadian teachers' and principals' beliefs about inclusive education as predictors of effective teaching in heterogeneous classrooms. Elementary School Journal, 98(3), 221-238.

Trujillo, F. (2007). Enseñar nuevas lenguas en la escuela: L1, L2, LE,..., NL. Revista de educación, 343, 71-91.

UNESCO (1994). Declaración de Salamanca y Marco de Acción para las necesidades eductivas especiales. Conferencia Mundial sobre Necesidades Educativas Especiales: Acceso y Calidad. UNESCO y Ministerio de Educación, España. París: UNESCO.

UNESCO (2005). Guidelines for inclusion: ensuring access to education for all. Paris: UNESCO.

Vila, I., Canal, I., Mayans, P., Perera, S., Serra, J.M. y Siqués, C. (2009). Las aulas de acogida de la educación primaria de Cataluña el curso 2005-2006: sus efectos sobre el conocimiento de catalán y la adaptación escolar. Infancia y Aprendizaje, 32 (3), 307-327.

Wilczenski, F. L. (1992). Measuring attitudes toward inclusive education. Psychology in the Schools, 29 (4), 306-312.

Wilczenski, F.L. (1995). Development of a scale to measure attitudes toward inclusive education. Educational and Psychological Measurement, 55 (2), 291-299.

Wyler, R.S, y Albarracín, D. (2005). Belief Formation, Organization, and Change: Cognitive and Motivational Influences. En Albarracín, D., Johnson, B.T. y Zanna, M.P(eds). The handbook of attitude (pp. 273-322). Mahwah, NJ: Erlbaum. 